10,11

\title{
Упругие свойства сжатых кристаллов инертных газов в модели деформируемых атомов
}

\author{
(C) Е.Е. Горбенко ${ }^{1}$, Е.П. Троицкая ${ }^{2}$, Е.А. Пилипенко \\ 1 Луганский национальный университет им. Тараса Шевченко, \\ Луганск, Украина \\ ${ }^{2}$ Донецкий физико-технический институт им. А.А. Галкина, \\ Донецк, Украина \\ ฯE-mail: pilipenko.katerina@mail.ru
}

(Поступила в Редакцию 10 мая 2016 г.)

\begin{abstract}
Упругие свойства сжатых кристаллов инертных газов $\mathrm{Ne}, \mathrm{Ar}, \mathrm{Kr}$ и Хе исследуются в модели деформируемых и поляризуемых атомов. Рассчитаны модули упругости Фукса второго порядка и их производные по давлению, а также коэффициент упругой анизотропии Зенера с учетом трехчастичного взаимодействия и деформации электронных оболочек квадрупольного типа в широком интервале давлений. Проведено сравнение с экспериментом и результатами других авторов. В Хе при сжатии 0.6 наблюдается обращение в нуль сдвигового модуля $B_{44}$, что соответствует ГЦК-ГПУ-переходу при $75 \mathrm{GPa}$.
\end{abstract}

DOI: 10.21883/FTT.2017.01.43962.167

\section{1. Введение}

Многочисленные теоретические [1-9] исследования модулей упругости второго порядка и других, связанных с ними, упругих свойств кристаллов инертных газов (КИГ) при высоких давлениях были проведены с использованием первопринципных методов и модельных потенциалов. Модули упругости третьего порядка для кристаллов были темой для обширного исследования в течение последних нескольких десятилетий и оценивались в ранних работах [10-14] в основном для ионных кристаллов.

Цикл работ [15-21] посвящен исследованию модулей упругости второго и высших порядков напряженных кристаллов с различным типом химической связи и симметрии. Часть результатов этих исследований представлена в монографии [22].

Большая величина отклонения от соотношения Коши, полученная в 2001 г. для $\mathrm{Ar}$ [23], указывает на то, что адекватное описание упругих свойств КИГ невозможно, используя только парные потенциалы. Первые попытки включить многочастичные эффекты в энергетические расчеты кристаллов инертных газов были проведены Аксильродом и Теллером [24].

Лехри и Верма [4] для изучения динамики решетки в КИГ разработали модель трехчастичного взаимодействия, включающую варьируемые индуцированные диполи. На базе этой модели в работе [5] они рассчитали модули упругости Браггера второго и третьего порядков для КИГ при $p=0$ в двух вариантах: 1) используя экспериментальное значение первой производной по давлению от объемного модуля $(d B / d p)$ и 2$)$ аппроксимируя трехчастичный потенциал экспоненциальной функцией, предложенной Кокрэном [25]. Остальные 4 параметра модели оценивались по 3 модулям упругости второго порядка и условию равновесия. Используя этот потенциал во втором варианте, включая вклад энергии нулевых колебаний (потенциал Лундквиста) в работе [26] были рассчитаны первая и вторая производные по давлению от объемного модуля и двух сдвиговых модулей для кристаллов ряда $\mathrm{Ne}-\mathrm{Xе} \mathrm{при} p=0$.

В работе [9] был использован также трехчастичный потенциал Лундквиста [27] для получения выражений модулей упругости Браггера второго и третьего порядков, производных первого порядка от объемного и сдвиговых модулей по давлению. Расчеты представлены для кристаллов $\mathrm{Ne}$ до $100 \mathrm{GPa}, \mathrm{Ar}$ до $75 \mathrm{GPa}, \mathrm{Kr}$ до $136 \mathrm{GPa}$ и Хе до $35.4 \mathrm{GPa}$.

Теория динамики решетки, развитая Толпыго на основе „первых принципов“ [28-31], успешно применялась для описания свойств щелочно-галоидных кристаллов, полупроводников и диэлектриков [32-37] при нулевом давлении.

Наиболее существенным в подходе Толпыго является выход за одноэлектронное приближение при построении адиабатического потенциала. А именно рассмотрение деформируемых электронных оболочек атомов учитывает отклик системы электронов кристалла на колебания ядер.

В серии работ (см. [38-42] и ссылки там) развита неэмпирическая версия модели Толпыго для изучения межатомного взаимодействия и динамической теории решеток ГЦК-кристаллов, подвергнутых всестороннему сжатию. В предыдущих работах [42-44] на основании общей теории, представленной в $[39,40]$ и модели разработанной в [41] проведено количественное исследование упругих модулей Бирча и соотношения Коши для всего ряда $\mathrm{Ne}-\mathrm{Xе} \mathrm{в} \mathrm{широком} \mathrm{интервале} \mathrm{давлений.} \mathrm{Показано,}$ что наблюдаемое отклонение от соотношения Коши $\delta(p)$ для каждого из кристаллов есть результат двух конкурирующих взаимодействий - многочастичного и квадрупольного, проявляющегося в квадрупольной деформации электронных оболочек атомов при смещениях 
ядер. Представленные $a b$ initio рассчитанные барические зависимости упругих модулей Бирча $\mathscr{B}_{i j}(p)$ и $\delta(p)$ хорошо согласуются с экспериментом.

Настоящая публикация продолжает цикл работ [39-44] посвященных неэмпирическим расчетам упругих свойств кристаллических $\mathrm{Ne}, \mathrm{Ar}, \mathrm{Kr}$ и Хе в актуальной области давлений - обращения в нуль фундаментальной щели. Расчеты проводились в модели деформируемых и поляризуемых атомов с учетом многочастичного взаимодействия. В работе исследуются упругие модули типа Фукса второго порядка $B_{i j}$, их производные по давлению $d B_{i k} / d p$ и коэффициент упругой анизотропии Зенера $A_{Z}$ для сжатых кристаллов ряда $\mathrm{Ne}-$ Хе. Рассчитываются некоторые модули Фукса третьего порядка $B_{i k l}$. Обсуждается проблема устойчивости ГЦК-решетки.

\section{2. Упругие свойства сжатых кристаллов}

При исследовании упругих свойств напряженного кристалла необходимо использовать теорию конечных деформаций $[45,46]$. При наличии напряжения различают три вида модулей упругости: коэффициенты разложения свободной энергии $C_{i k l . . .}$ (модули типа Браггера), коэффициенты пропорциональности в законе Гука в напряженном кристалле $\mathscr{B}_{i k l . . .}$ (модули Бирча) и коэффициенты распространения звука в напряженном кристалле $A_{i k l \ldots}$ Обычно при этом используется в качестве параметров разложения лагранжевый тензор дисторсии $u_{\alpha \beta}$. Модули Браггера $C_{\alpha \beta \gamma}$ общеприняты, однако иногда удобнее использовать параметры деформации $\gamma_{i}$, которые имеют наглядный физический смысл.

Параметры $\gamma_{i}$ вводятся так, чтобы они обращались в нуль при исчезновении деформации, а соответствующими им деформациями являются: всестороннее сжатие $\left(\gamma_{1}\right)$, одноосные сжатия $\left(\gamma_{2}, \gamma_{3}\right)$ и сдвиги $\left(\gamma_{4}, \gamma_{5}, \gamma_{6}\right)$. Если имеется несколько атомов в элементарной ячейке, то параметры $\gamma_{7}, \gamma_{8}, \ldots$ описывают относительные смещения этих атомов при деформации кристалла. По определению для одноатомного кристалла тензор дисторсии $u_{\alpha \beta}$ выражается через $\gamma_{i}$ следующим образом (см., например, [22]):

$$
\begin{gathered}
1+u_{11}=\left(1+\gamma_{1}\right)^{1 / 3}\left(1+\gamma_{2}\right)^{-1 / 3}\left(1+\gamma_{3}\right)^{-1 / 2}=f_{1}\left(\gamma_{1}, \gamma_{2}, \gamma_{3}\right), \\
1+u_{22}=\left(1+\gamma_{1}\right)^{1 / 3}\left(1+\gamma_{2}\right)^{-1 / 3}\left(1+\gamma_{3}\right)^{1 / 2}=f_{2}\left(\gamma_{1}, \gamma_{2}, \gamma_{3}\right), \\
1+u_{33}=\left(1+\gamma_{1}\right)^{1 / 3}\left(1+\gamma_{2}\right)^{2 / 3}=f_{3}\left(\gamma_{1}, \gamma_{2}\right), \\
u_{23}=\gamma_{4}, \quad u_{13}=\gamma_{5}, \quad u_{12}=\gamma_{6}, \quad u_{32}=u_{31}=u_{21}=0 .
\end{gathered}
$$

Производная свободной энергии $F(\gamma)$ по параметрам $\gamma_{1}-\gamma_{6}$ определяет упругие модули типа Фукса, физически наглядные при больших деформациях

$$
B_{i \ldots l}=\frac{1}{\Omega}\left(\frac{\partial^{n} F(\gamma)}{\partial \gamma_{i} \ldots \partial \gamma_{l}}\right)_{\gamma=0} .
$$

В дальнейшем поведение сжатого кристалла будем описывать этими модулями второго порядка $B_{i k}$, их производными по давлению $d B_{i k} / d p$ и соответствующими модулями третьего порядка $B_{i k l}$.

Установим связь между модулями второго порядка Фукса $B_{i k}$, Браггера $C_{i k}$ и Бирча $\mathscr{B}_{i j}$ в напряженном кристалле $(p \neq 0)$.

$$
\begin{aligned}
B_{11}=\frac{1}{3}\left(C_{11}+2 C_{12}\right)+\frac{1}{3} p ; & C_{11}=B_{11}+\frac{4}{3} B_{33}+p \\
B_{33}=\frac{1}{2}\left(C_{11}-C_{12}\right)-p ; & C_{12}=B_{11}-\frac{2}{3} B_{33}-p \\
B_{44}=C_{44}-p ; & C_{44}=B_{44}+p
\end{aligned}
$$

Объемный модуль (bulk modul $B$ )

$$
B=\left(C_{11}+2 C_{12}\right) / 3=B_{11}-\frac{1}{3} p .
$$

Сдвиговый модуль

$$
C^{\prime}=\left(C_{11}-C_{12}\right) / 2=B_{33}+p .
$$

А модули Бирча выражаются как

$$
\begin{gathered}
\mathscr{B}_{\alpha \beta \gamma \vartheta}=C_{\alpha \beta \gamma \vartheta}-p\left(\delta_{\alpha \gamma} \delta_{\beta \vartheta}+\delta_{\alpha \vartheta} \delta_{\beta \gamma}-\delta_{\alpha \beta} \delta_{\gamma \vartheta}\right), \\
\mathscr{B}_{11}=B_{11}+\frac{4}{3} B_{33}=C_{11}-p, \\
\mathscr{B}_{12}=B_{11}-\frac{2}{3} B_{33}=C_{12}+p, \\
\mathscr{B}_{44}=B_{44}=C_{44}-p .
\end{gathered}
$$

Модули типа Браггера $C_{i j}$ зависят от давления $p$, а $B_{i k}$ можно скомпоновать только из модулей Бирча $\mathscr{B}_{i j}$, которые в случае кубического кристалла СІ непосредственно измеряются в ультразвуковых экспериментах. Игнорирование этого обстоятельства приводит к путанице численных значений модулей упругости напряженных кристаллов.

\section{3. Результаты расчета модулей упругости Фукса второго порядка}

В предыдущих работах [42-44] для всего ряда сжатых КИГ были рассчитаны барические зависимости упругих модулей Бирча $\mathscr{B}_{i j}$, и отклонений от соотношения Коши $\delta(p)$ с учетом многочастичного взаимодействия и деформации электронных оболочек в квадрупольном приближении. Сравнение теоретических величин $\delta(p)$ с экспериментальными показало адекватность построенной теории и модели расчета параметров [39-41].

Запишем модули Фукса второго порядка $B_{i j}$ через полученные параметры в виде

$$
\begin{gathered}
B_{11}=\frac{e^{2}}{6 a^{4}}\left[2 G-H-2 F+2 E-V_{t}-2.710107 D\right] ; \\
B_{33}=\frac{e^{2}}{8 a^{4}}\left[G+4 H+8 F+2 E-2 V_{q}+V_{t}-0.231934 D\right] ; \\
B_{44}=\frac{e^{2}}{4 a^{4}}\left[G+2 H+4 F-8 V_{q}+V_{t}-0.52494 D\right] .
\end{gathered}
$$

Здесь $H=H_{0}+\delta H, G=G_{0}+\delta G, H_{0}\left(r_{0}\right)$ и $G_{0}\left(r_{0}\right)$ являются первой и второй производными парно- 

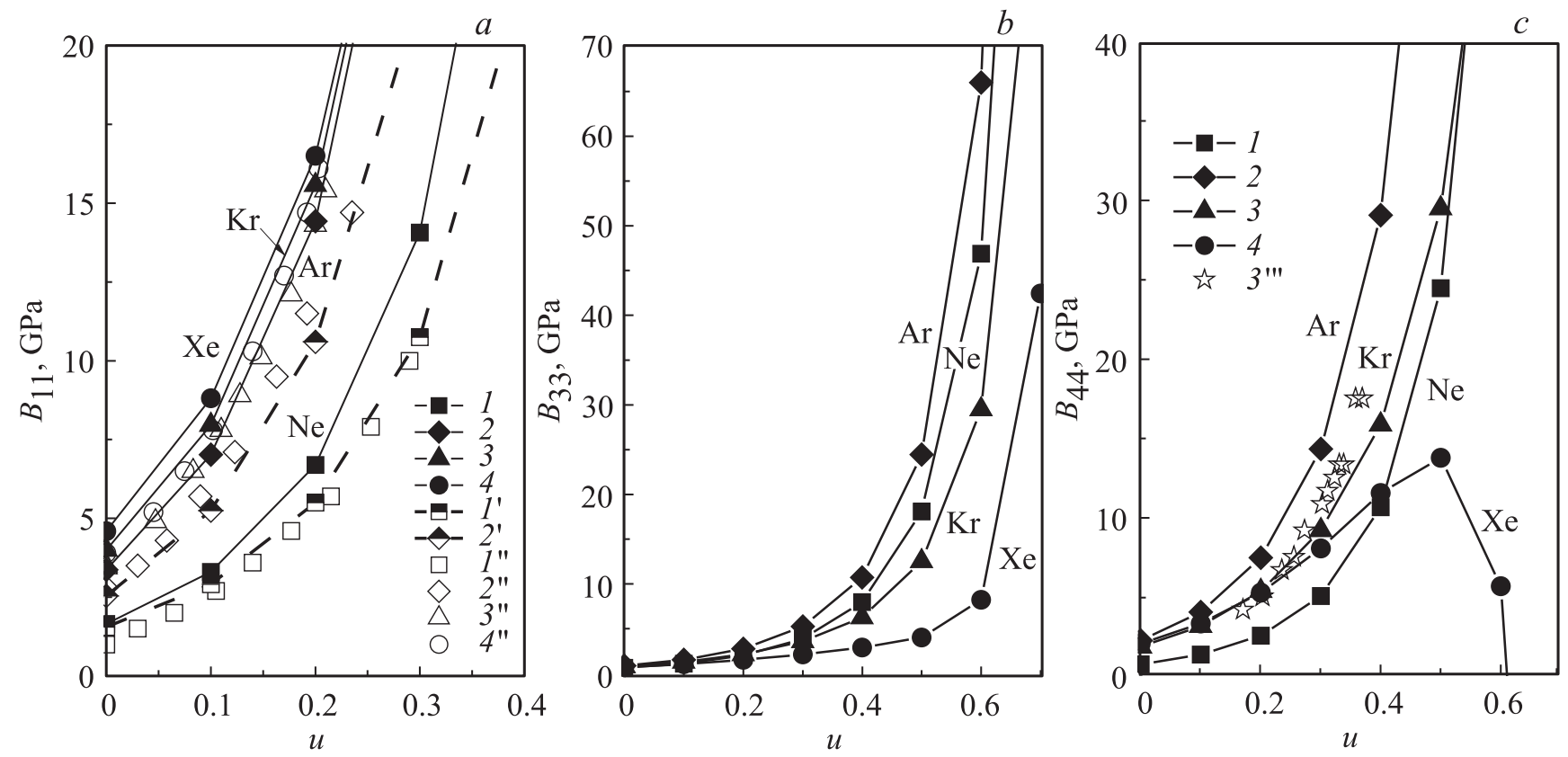

Рис. 1. Барические зависимости модулей упругости Фукса второго порядка $B_{11}(a), B_{33}(b), B_{44}(c)$ для $\mathrm{Ne}(1), \operatorname{Ar}(2), \operatorname{Kr}(3)$ и Хе (4). 1-4 - расчет $B_{11}$ настоящей работы с учетом трехчастичного и квадрупольного взаимодействий $B_{11}=B_{11}^{0}+B_{11}^{t}$ $+B_{11}^{q}(13)$, где $B_{11}^{0}$ рассчитывается в модели M3; $1^{\prime}-$ расчеты на основе $B_{11}^{0}$ в модели M5 для Ne; $2^{\prime}-$ расчеты на основе $B_{11}^{0}$ в модели М1 для Ar; $1^{\prime \prime}-4^{\prime \prime}-$ эксперимент [48]; $3^{\prime \prime \prime}$ - эксперимент [49] для $\mathrm{Kr}$.

го короткодействующего потенциала отталкивания $V_{s r}=E^{(0)}+W_{2}$ для равновесных расстояний первых соседей $\left(r_{0}=a \sqrt{2}\right) ; \delta H$ и $\delta G-$ трехчастичные поправки, приводящие к нецентральности парного взаимодействия; $F, E$ - короткодействующие парные силы между вторыми соседями $F=H_{0}(2 a), E=G_{0}(2 a) ; V_{t}$ и $V_{q}$ - параметры трехчастичного и квадрупольного взаимодействия соответственно; $D$ определяет взаимодействие Ван-дерВаальса. (Таблицу рассчитанных параметров для различных сжатий см., например, в [44]).

Модули Фукса (12) можно представить как

$$
B_{i j}=B_{i j}^{(0)}+B_{i j}^{t}+B_{i j}^{q}
$$

$B_{11}^{t}=\frac{1}{3} K(p)\left[2 \delta G-\delta H-V_{t}\right], \quad B_{11}^{q}=0, \quad K(p)=\frac{e^{2}}{2 a^{4}}$,

$$
B_{33}^{t}=\frac{1}{4} K(p)\left[\delta G+4 \delta H+V_{t}\right], \quad B_{33}^{q}=K(p)\left[-\frac{1}{2} V_{q}\right],
$$$$
B_{44}^{t}=\frac{1}{2} K(p)\left[\delta G+2 \delta H+V_{t}\right], \quad B_{44}^{q}=K(p)\left[-4 V_{q}\right],
$$

$B_{i j}^{0}$ - рассчитанные нами ранее модули упругости Фукса с парным потенциалом в моделях М1, M3 и M5 [21]. В модели М5 короткодействующая часть потенциала отталкивания рассчитана по точной формуле для квадратичной поправки $W_{2}$ (см. [47]) $V_{s r}=E^{(0)}+W_{2}\left(S^{n}\right)$, в других моделях М3 и M1 $W_{2}$ рассчитывается в приближении $S^{2}$. Кроме того, в моделях М3 и М5 переопределена константа Ван-дер-Ваальса $C \rightarrow C(1-A \exp (-\beta r))$ и учтены в расчетах короткодействующих сил „вторые“ соседи [21]. В самой простой модели М1 $A=\beta=0$ и учтены только первые соседи.

На рис. $1, a-c[48,49]$ приведены результаты расчетов модулей упругости Фукса $B_{i j}$ с учетом трехчастичного и квадрупольного взаимодействий в зависимости от сжатия $u=\Delta V / V_{0}\left(\Delta V=V_{0}-V(p)\right.$, где $V_{0}$ - объем при $p=0)$ для $\mathrm{Ne}, \mathrm{Ar}, \mathrm{Kr}$ и Хе. Как видно из рис. 1, $a$, согласие теоретической величины $B_{11}$ и экспериментальной [48] хорошее и зависит от выбора модели расчета парного потенциала. Так, для $\mathrm{Ne}$ в качестве базовой необходимо использовать модель M5 [21], для $\mathrm{Ar}-$ M1, а для тяжелых кристаллов инертных газов $\mathrm{Kr}$ и Хе - M3 [21]. В отклонениях теории (расчет на основе моделей М3 и М5) от эксперимента заметна определенная тенденция: теоретический модуль $B_{11}$ ложится несколько выше экспериментальных кривых для всего ряда $\mathrm{Ne}-\mathrm{Xe}$.

Зависимость сдвигового модуля $B_{33}$ от сжатия имеет тот же характер, что и $B_{11}(u)$, хотя монотонность в ряду $\mathrm{Ne}, \mathrm{Ar}, \mathrm{Kr}$, Хе нарушается (рис. $1, b$ ).

$\mathrm{B}$ Хе при сжатиях $u=0.6$ сдвиговый модуль $B_{44}$ обращается в нуль. Это указывает на появление абсолютной неустойчивости и необходимости фазового перехода. Действительно такой переход был экспериментально обнаружен в [50]. Это переход из промежуточной плотноупакованной в ГПУ-фазу при $75 \mathrm{GPa}$ непосредственно перед металлизацией, происходящей при $u=0.65(132 \mathrm{GPa})[51]$.

Отметим, что при высоком давлении учет трехчастичного и квадрупольного взаимодействий приводит к нару- 
Производные по давлению от объемного $d B / d p$ и сдвиговых $d C^{\prime} / d p, d C_{44} / d p$ модулей упругости и модули упругости Фукса третьего порядка $B_{111}, B_{133}, B_{144}$ для кристаллов ряда $\mathrm{Ne}-$ Хе при нулевом давлении

\begin{tabular}{|c|c|c|c|c|c|c|c|c|c|c|}
\hline КИГ & Теория & $a, \AA$ & $\begin{array}{c}d B / d p \\
\text { (эксп. [52]) }\end{array}$ & $d B / d p$ & $\gamma, \%$ & $d C^{\prime} d p$ & $d C_{44} / d p$ & $B_{111}$ & $B_{133}$ & $B_{144}$ \\
\hline \multirow[t]{3}{*}{$\mathrm{Ne}$} & $\begin{array}{l}\text { Настоящий } \\
\text { расчет }\end{array}$ & 2.231 & \multirow[t]{3}{*}{7} & 5.862 & 16.3 & 2.804 & 3.295 & -11.88 & -2.304 & -3.548 \\
\hline & $\begin{array}{l}\text { Лехри и } \\
\text { Верма [5] }\end{array}$ & 2.227 & & $\begin{array}{c}10.2 \\
-\end{array}$ & 45.7 & $\begin{array}{c}2.63 \\
(2.23)\end{array}$ & $\begin{array}{c}8.45 \\
(7.65)\end{array}$ & $\begin{array}{l}-12.475 \\
(-8.85)\end{array}$ & $\begin{array}{l}-2.422 \\
(-1.06)\end{array}$ & $\begin{array}{l}-7.131 \\
(-6.23)\end{array}$ \\
\hline & $\begin{array}{l}\text { Гаупта } \\
\text { и Гоял [9] }\end{array}$ & 2.227 & & 6.66 & 4.8 & 1.91 & 6.10 & - & - & - \\
\hline \multirow[t]{3}{*}{$\mathrm{Ar}$} & $\begin{array}{l}\text { Настоящий } \\
\text { расчет }\end{array}$ & 2.656 & \multirow[t]{3}{*}{7} & 7.247 & 3.5 & 2.383 & 4.774 & -28.91 & -3.747 & -11.97 \\
\hline & $\begin{array}{l}\text { Лехри и } \\
\text { Верма [5] }\end{array}$ & 2.656 & & $\begin{array}{c}8.9 \\
-\end{array}$ & 27.1 & $\begin{array}{c}2.03 \\
(1.79)\end{array}$ & $\begin{array}{c}7.02 \\
(6.53)\end{array}$ & $\begin{array}{l}-29.822 \\
(-24.01)\end{array}$ & $\begin{array}{c}-5.167 \\
(-3.057)\end{array}$ & $\begin{array}{l}-15.617 \\
(-14.16)\end{array}$ \\
\hline & $\begin{array}{l}\text { Гаупта и } \\
\text { Гоял [9] }\end{array}$ & 2.656 & & 7.74 & 10.6 & 1.8 & 5.81 & - & - & - \\
\hline \multirow[t]{3}{*}{$\mathrm{Kr}$} & $\begin{array}{l}\text { Настоящий } \\
\text { расчет }\end{array}$ & 2.824 & \multirow[t]{3}{*}{7} & 7.16 & 2.3 & 1.953 & 3.49 & -33.956 & -2.966 & -9342 \\
\hline & $\begin{array}{l}\text { Лехри и } \\
\text { Верма [5] }\end{array}$ & 3.323 & & $\begin{array}{c}9.9 \\
-\end{array}$ & 41.4 & $\begin{array}{c}2.43 \\
(2.06)\end{array}$ & $\begin{array}{c}7.61 \\
(6.89)\end{array}$ & $\begin{array}{l}-39.309 \\
(-28.81)\end{array}$ & $\begin{array}{c}-7.699 \\
(-3.597)\end{array}$ & $\begin{array}{l}-21.337 \\
(-18.71)\end{array}$ \\
\hline & $\begin{array}{l}\text { Гаупта и } \\
\text { Гоял }[9]\end{array}$ & 2.823 & & 9.27 & 32.4 & 2.24 & 6.63 & - & - & - \\
\hline \multirow[t]{3}{*}{$\mathrm{Xe}$} & $\begin{array}{l}\text { Настоящий } \\
\text { расчет }\end{array}$ & 3.063 & \multirow[t]{3}{*}{7} & 7.162 & 2.3 & 1.625 & 3.347 & -39.002 & -2.116 & -10.095 \\
\hline & $\begin{array}{l}\text { Лехри и } \\
\text { Верма [5] }\end{array}$ & 3.065 & & $\begin{array}{c}9.7 \\
-\end{array}$ & 38.6 & $\begin{array}{c}2.29 \\
(1.93)\end{array}$ & $\begin{array}{c}7.72 \\
(7.01)\end{array}$ & $\begin{array}{l}-39.307 \\
(-29.04)\end{array}$ & $\begin{array}{l}-7.380 \\
(-3.53)\end{array}$ & $\begin{array}{l}-21.273 \\
(-18.72)\end{array}$ \\
\hline & $\begin{array}{l}\text { Гаупта и } \\
\text { Гоял }[9]\end{array}$ & 3.065 & & 8.48 & 21.1 & 2.14 & 6.54 & - & - & - \\
\hline
\end{tabular}

Пр имечан и е. В скобках приведены результаты Лехри и Верма [5], использующие в расчетах экспериментальное значение величины $d B / d p[52]$. Относительная погрешность $\gamma=\left|\frac{(d B / d p)^{\exp }-(d B / d p)^{\text {theor }}}{(d B / d p)^{\exp }}\right| \cdot 100 \%$.

шению линейной барической зависимости, характерной для $\mathscr{B}_{i j}^{0}(p)$ и $B_{i j}^{0}[21,42]$.

\section{4. Модули упругости Фукса третьего порядка}

Модули упругости высших порядков содержат более высокие (чем вторая) пространственные производные энергии связи. Из-за резкого изменения межатомного потенциала с расстоянием модули упругости Фукса третьего порядка $B_{i k l}$ значительно больше модулей упругости второго порядка $B_{i k}$ и могут служить хорошим тестом применимости той или иной теории.

В случае кубического кристалла СI существует один модуль первого порядка $\left(B_{1}=-p\right)$, три модуля второго порядка $B_{i k}$ и шесть модулей третьего порядка $B_{i k l}$ (см., например, [22], С. 51). Три модуля $B_{111}, B_{133}, B_{144}$ можно найти из измерений (расчетов) величин $d B_{i k} / d p$ при $p=0$, с которыми они связаны соотношениями

$$
\begin{gathered}
B_{111}=-B\left(\frac{d B_{11}}{d p}+1\right) \\
B_{133}=C^{\prime}-B \frac{d B_{33}}{d p} \\
B_{144}=\frac{1}{3} C_{44}-B \frac{d B_{44}}{d p}
\end{gathered}
$$

где $B$ и $C^{\prime}$ определены формулами (6), (7);

$\frac{d B_{11}}{d p}=\frac{d B}{d p}+\frac{1}{3} ; \quad \frac{d B_{33}}{d p}=\frac{d C^{\prime}}{d p}-1 ; \quad \frac{d B_{44}}{d p}=\frac{d C_{44}}{d p}-1$.

В таблице приведены рассчитанные нами в модели деформированных атомов с учетом трехчастичного взаимодействия первые производные по давлению от объемного и сдвиговых модулей $d B_{i k} / d p$ и модули 

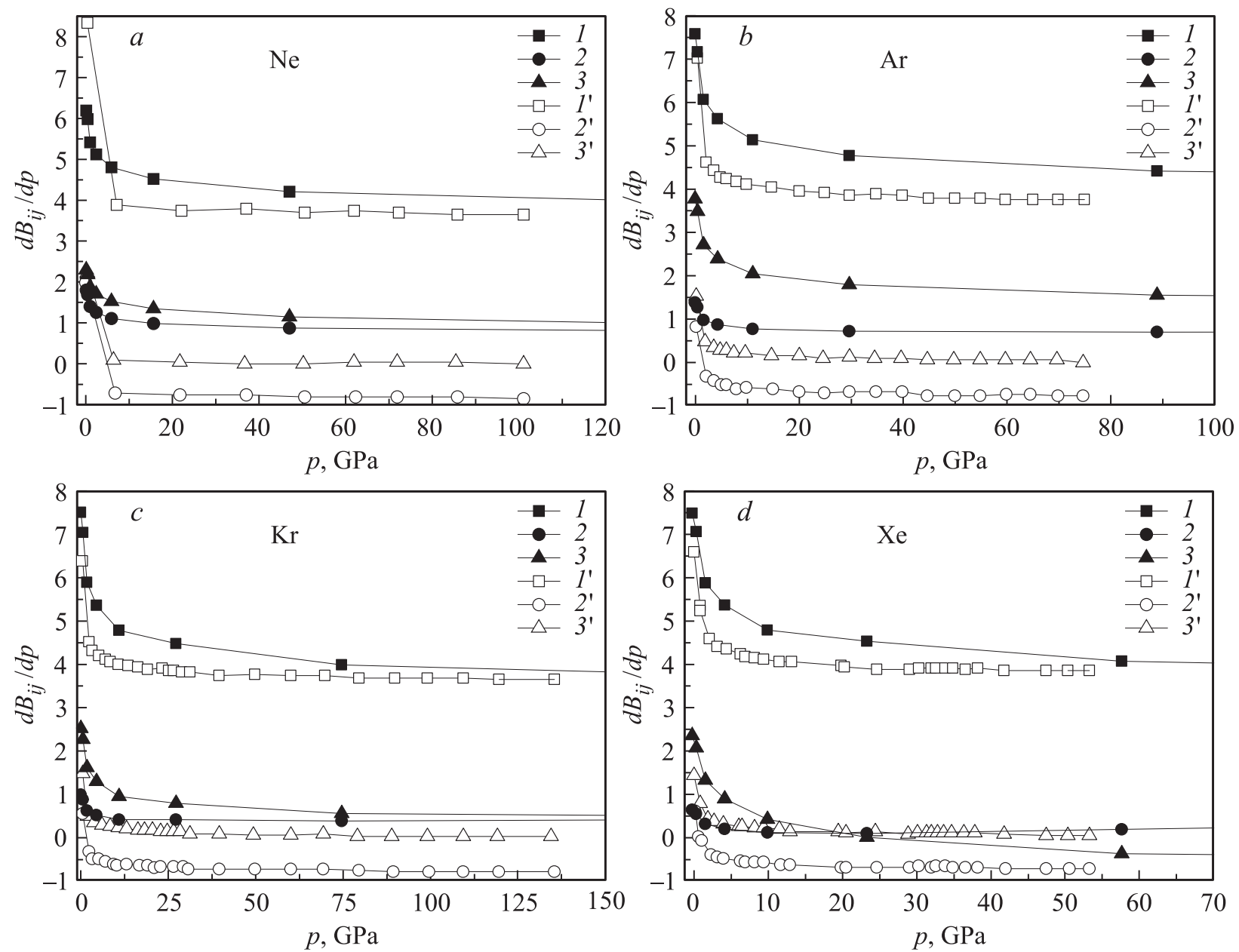

Рис. 2. Барические зависимости производных по давлению от модулей упругости Фукса второго порядка $d B_{11} / d q(1), d B_{33} / d p(2)$, $d B_{44} / d p(3)$ для $\mathrm{Ne}(a), \operatorname{Ar}(b), \operatorname{Kr}(c)$ и $\mathrm{Xe}(d) .1-3$ - расчет $d B_{i j} / d p$ настоящей работы; $1^{\prime}-3^{\prime}-$ расчет $d B_{i j} / d p$ по формуле (15), с использованием $d B / d p, d C^{\prime} / d p, d C_{44} / d p$, полученных в работе [5].

упругости Фукса третьего порядка $B_{i k l}(14)$ при $p=0$, результаты других авторов [5,9] и эксперимент [52].

Чтобы сравнить наши результаты расчетов $B_{i k l}$ с результатами расчетов $C_{i k l}$ из работы [5], мы воспользовались следующими соотношениями, справедливыми при $p=0$ (см., например, [22], С. 51)

$$
\begin{gathered}
B_{111}=-B+\frac{1}{9}\left(C_{111}+6 C_{112}+2 C_{123}\right) ; \\
B_{133}=B+\frac{4}{3} C^{\prime}+\frac{1}{6}\left(C_{111}+C_{123}\right) ; \\
B_{144}=B+\frac{2}{3} C_{44}+\frac{1}{3}\left(C_{144}+2 C_{166}\right) .
\end{gathered}
$$

Как видно из таблицы, наш расчет $d B / d p$ очень хорошо согласуется с экспериментом [52] для $\mathrm{Ar}-\mathrm{Xe}$ (погрешность $\sim 3 \%$ ) и несколько хуже - для $\mathrm{Ne}$. Peзультаты расчетов [9] и [5] на основе эмпирического потенциала Лундквиста согласуются с экспериментом [52] значительно хуже, чем наши. Для производных по давлению от двух сдвиговых модулей экспериментальных значений, к сожалению, нет, результаты наших расчетов значительно отличаются от результатов других авторов, особенно для $d C_{44} / d p$. Модули упругости третьего порядка $B_{i k l}$, определяемые производными по давлению от модулей упругости второго порядка $d B_{i k} / d p$ демонстрируют то же поведение. Лучше всего наши расчеты $B_{111}$ согласуются (с точностью до 5\%) с расчетами Лехри и Верма [5], выполненными во втором варианте, описанном во введении. Исключение составляет только $\mathrm{Kr}$, для которого относительная погрешность $\gamma^{\mathrm{Kr}}=14 \%$. Наш расчет $B_{133}$ в легких кристаллах ложится в интервал между расчетами [5], полученными в двух различных моделях, а в $\mathrm{Kr}$ и Хе он несколько ниже, как и в случае $B_{144}$ для всех КИГ.

На рис. 2, $a-d$ представлены величины $d B_{i k} / d p$ как функции давления для $\mathrm{Ne}, \mathrm{Ar}, \mathrm{Kr}, \mathrm{Xe}$. Для сравнения мы привели соответствующие расчеты $d B_{i k} / d p$ через $d C_{i k} / d p$ (15) полученные в работе [9] на основе трехчастичного потенциала Лундквиста [27]. В обоих случаях все три величины $d B_{i k} / d p$ уменьшаются с ростом давления до $15-25 \mathrm{GPa}$, которое соответствует сжатию 

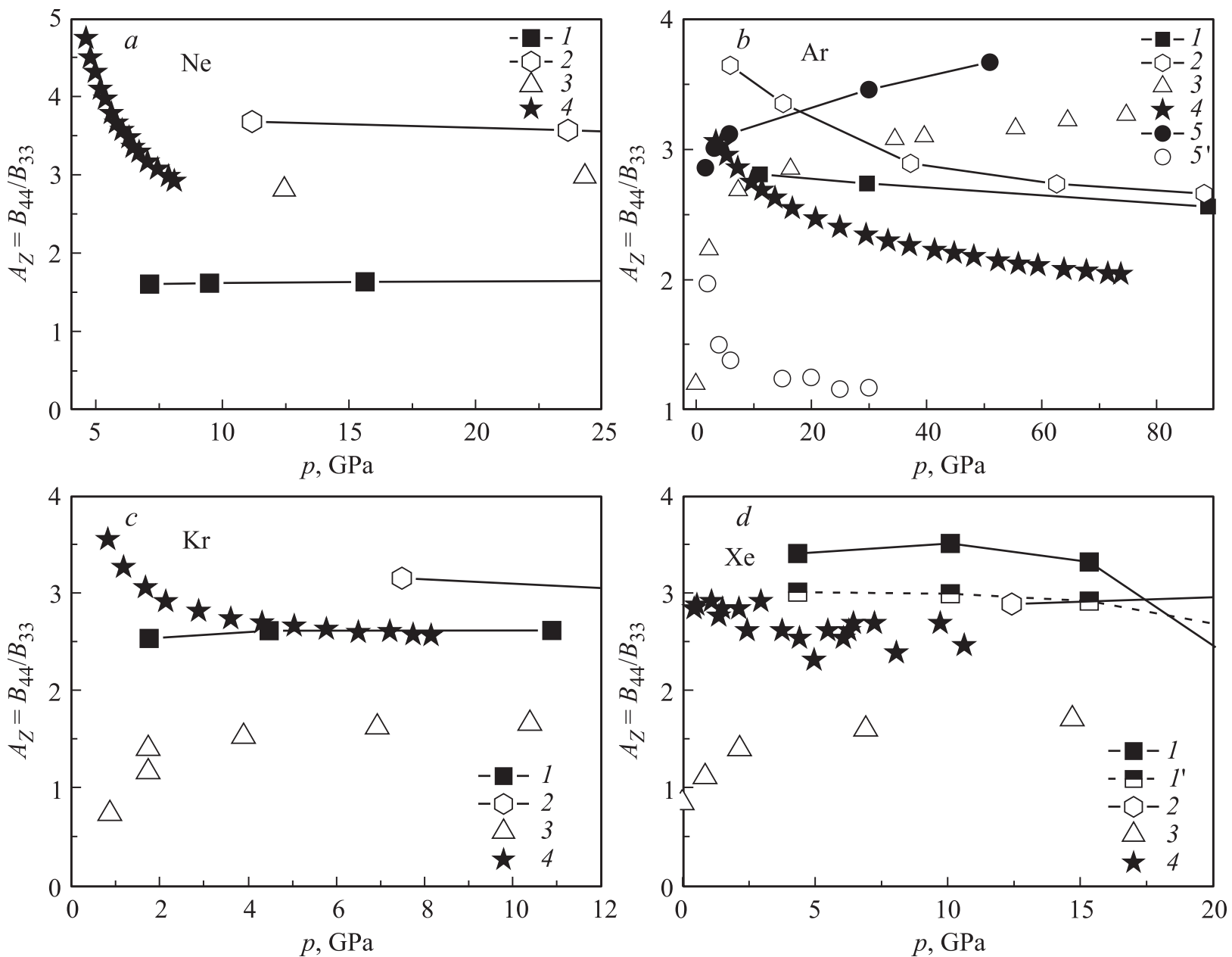

Рис. 3. Барические зависимости коэффициента упругой анизотропии Зенера $A_{Z}(p)$ для $\mathrm{Ne}(a), \operatorname{Ar}(b), \operatorname{Kr}(c)$ и $\mathrm{Xe}(d)$. $1-$ расчет настоящей работы с учетом трехчастичного и квадрупольного взаимодействий; $1^{\prime}$ - наш расчет $A_{Z}(p)$ в Хе на основе парного потенциала; 2 - расчет в DFT [3]; 3 - расчет $A_{Z}$, полученный в работе [9]; 4 - эксперимент для $\mathrm{Ne}$ [57], $\mathrm{Ar}$ [23], $\mathrm{Kr}$ [49], $\mathrm{Xe}$ [59]; 5 - теоретический расчет $A_{Z}(p)$, полученный в работе [58]; $5^{\prime}$ - эксперимент [58].

$u=0.4-0.5$ для всего ряда $\mathrm{Ne}-\mathrm{Xe} . \mathrm{B}$ интервале сжатий с $u=0.5$ до $u=0.7$, последнее соответствует давлению $p \approx 180 \mathrm{GPa}$ в $\mathrm{Ne}, p \approx 330 \mathrm{GPa}$ в $\mathrm{Ar}, p \approx 250 \mathrm{GPa}$ в $\mathrm{Kr}$ и до $u=0.6(p \approx 60 \mathrm{GPa})$ в Хе наблюдается слабая барическая зависимость $d B_{i k} / d p$.

Как видно из рис. 2, лучше всего согласуются наши расчеты и расчеты [9] барических зависимостей $d B_{11} / d p$ и $d B_{44} / d p$, хуже всего $d B_{33} / d p$. Причем в легких кристаллах расхождение значительнее, чем в тяжелых.

\section{5. Упругая анизотропия Зенера}

Анизотропия является следствием того, что в структуре кристалла в разных направлениях различны расстояния и силы связи между атомами и проявляется тем сильнее, чем ниже симметрия кристаллов. Коэффициент Зенера $A_{Z}$, является наиболее известной мерой упругой анизотропии, который применяется для кубического монокристалла. Впервые это понятие было введено в
1947 г. при исследовании $\beta$-латуни [53]. С течением времени в литературе был описан широкий спектр единиц измерений упругой анизотропии. Авторы наряду с коэффициентом Зенера для кубических кристаллов использовали различные комбинации упругих постоянных, для кристаллов с симметрией низшего порядка [54-56]. Количественно упругую анизотропию оценивают по максимальному различию модулей упругости. Так коэффициент упругой анизотропии Зенера для кристаллов с кубической симметрией определяется как отношение двух сдвиговых модулей Фукса $A_{Z}=B_{44} / B_{33}, A_{Z}=1$, если материал изотропный. Модуль $\mathrm{B}_{44}$ относится к сдвигу всей плоскости (100) в произвольном направлении, а $B_{33}-$ к сдвигу через плоскости (110) в направлении [1̄0] [53].

На рис. $3, a-d[3,9,23,49,57-59]$ упругая анизотропия Зенера $A_{Z}$ представлена как функция давления для $\mathrm{Ne}, \mathrm{Ar}, \mathrm{Kr}$ и Хе. Для экспериментальных данных $\mathrm{Ne}$ характерно быстрое уменьшение от 4.9 при $p=5 \mathrm{GPa}$ до 3.6 при $p=7 \mathrm{GPa}$ [57]. Как видно из рис. $3, a$, наш 
расчет $A_{Z}$ для $\mathrm{Ne}$ расположен ниже экспериментальной кривой [57] и расчетов $A_{Z}$ других авторов [3,9].

Экспериментальная зависимость коэффициента Зенера $A_{Z}$ от давления была определена до $30 \mathrm{GPa}[58]$ и $70 \mathrm{GPa}[23]$ для $\mathrm{Ar}$, и до $8 \mathrm{GPa}$ [49] для $\mathrm{Kr}$. На рис. 3, b, c видно, что полученные нами значения расположены между 2.8 и 2.5 и с ростом давления стремятся к изотропному пределу $A_{Z}=1$. Как можно увидеть, наши результаты для $\mathrm{Ar}$ и $\mathrm{Kr}$ согласуются с экспериментом $[23,49]$ и расчетами $A_{Z}$ в теории функционала плотности (density functional theory - DFT) [3] довольно хорошо. На примере Ar хорошо видно различие в экспериментальных данных, полученных группами авторов Шимизу и др. [23] и Гримсдич и др. [58]. Это различие объясняется тем, что авторы $[58,60]$ измеряли барическую зависимость продольной акустической скорости для произвольных неизвестных направлений в монокристаллах $\mathrm{Ar}$ и $\mathrm{Kr}$ без определения ориентации кристалла. В отличие от них Шимизу с коллегами $[23,49,57,59]$ при измерении модулей упругости проводили идентификацию ориентации кристалла для каждого давления.

Как видно на рис. $3, d$, наш расчет для Хе, основанный на парном потенциале, находится в хорошем согласии с экспериментом [59], и соответствует классической зависимости $A_{Z}(p)$. Рассчитанные величины $A_{Z}$ с учетом трехчастичного и квадрупольного взаимодействий, расположены несколько выше, чем экспериментальные данные, и начиная с $15 \mathrm{GPa}$ быстро стремятся к нулю, демонстрируя неустойчивость ГЦК-фазы. В работе [50] при рентгеновском исследовании сжатого Хе, было показано, что ГЦК-Хе начиная с $p=14 \mathrm{GPa}$, превращается в промежуточную плотноупакованную фазу, а затем полностью преобразуется в ГПУ структуру при $75 \mathrm{GPa}$.

Как можно увидеть из рис. $3, a-d$ общим для экспериментальных результатов всего ряда $\mathrm{Ne}-\mathrm{Xe}[23,49,57,58,59]$, является уменьшение коэффициента упругой анизотропии Зенера $A_{Z}$ с ростом давления и стремление к изотропному пределу $A_{Z}=1$. Однако, как отмечается в работах $[9,61]$ для КИГ характерно увеличение ими рассчитанной упругой анизотропии с ростом давления, что противоречит эксперименту. Результаты наших расчетов коэффициента упругой анизотропии Зенера в широком интервале давления (сжатия) представлены в [62]. Полученные нами величины $A_{Z}$ постепенно увеличиваются до определенного сжатия, подобно другим теоретическим работам [9,58,61]. Однако начиная с сжатий $u=0.6$ $(p \approx 50 \mathrm{GPa})$ в $\mathrm{Ne}, u=0.5(p \approx 30 \mathrm{GPa})$ в $\mathrm{Ar}, u=0.4$ $(p \approx 10 \mathrm{GPa})$ в $\mathrm{Kr}$ и $u=0.5(p \approx 15 \mathrm{GPa})$ в Хе наблюдается уменьшение $A_{Z}$ и стремление его к изотропному пределу $A_{Z}=1$, что соответствует эксперименту.

\section{6. Заключение}

При анализе упругих свойств сжатых кристаллов ряда $\mathrm{Ne}-\mathrm{Xе} \mathrm{мы} \mathrm{уделили} \mathrm{довольно} \mathrm{большое} \mathrm{внима-}$ ние сравнению наших расчетов барической зависимости упругой анизотропии Зенера $A_{Z}(p)$ с экспериментом, хотя, на наш взгляд, $A_{Z}(p)$ менее информативен, чем отклонение от соотношения Коши $\delta(p)$, поскольку в случае $A_{Z}(p)$ роль трехчастичного взаимодействия и эффектов деформации электронных оболочек маскируются более сильным парным взаимодействием. Анализ результатов расчетов барических зависимостей трех модулей упругости второго порядка $\mathscr{B}_{i j}(p)$ (или их комбинаций), отклонения от соотношения Коши $\delta(p)$ и упругая анизотропия Зенера $A_{Z}(p)$, выполненных в теории функционала плотности [3] или на основе эмпирических потенциалов [7-9,61], как правило, показывает количественное согласие с экспериментом для модулей упругости $\mathscr{B}_{i j}(p)$ лучше, чем у нас, однако для $\delta(p)$ и $A_{Z}(p)$ нет даже качественного согласия в отличие от наших расчетов $[42,44]$.

В работе [5] настораживает большое различие в результатах расчетов $d C_{i k} / d p$ и $C_{i k l}$, выполненных в двух вариантах. Нам ближе второй вариант, в котором параметр трехчастичного взаимодействия определяется по экспоненциальной формуле Кокрэна [25]. Во всяком случае, модули $B_{111}$ близки к нашим, хотя их первая производная по давлению от объемного модуля $d B / d p$ плохо согласуется с экспериментом. Хорошее согласие, полученных нами, величин $d B / d p$ с имеющимися экспериментальными данными при $p=0$, адекватное описание $A_{Z}(p)$, и в особенности $\delta(p)$, позволяет сделать вывод в пользу предсказательной ценности расчетов упругих, динамических и термодинамических свойств сжатых КИГ в модели деформируемых и поляризуемых атомов на основании неэмпирического короткодействующего потенциала.

Таким образом, межатомный потенциал, полученный в квантово-механической модели деформируемых и поляризуемых атомов (модель Толпыго) с учетом трехчастичного взаимодействия и деформации электронных оболочек атомов отражает все существенные черты поведения атомарных криокристаллов под давлением.

Статья посвящена светлой памяти Кирилла Борисовича Толпыго, столетие со дня рождения которого исполнилось в 2016 году.

\section{Список литературы}

[1] T. Tsuchiya, J. Kawamura. Chem. Phys. 114, 10086 (2001).

[2] T. Tsuchiya, J. Kawamura. Chem. Phys. 116, 2121 (2002).

[3] T. Tsuchiya, J. Kawamura. Chem. Phys, 117, 5859 (2002).

[4] S. Lehri, M.P. Verma. Phys. Status solidi B 92, 363 (1979).

[5] S. Lehri, M.P. Verma. Phys. Status Solidi B 98, 789 (1980).

[6] A.L. Karasevskii, W.B. Holzapfel. Phys. Rev. 67, 224301 (2003).

[7] M. Aoki, T. Kurokawa. J. Phys. Condens. Matter 19, 236228 (2007).

[8] E. Pechenic, I. Kelson, G. Makov. Phys. Rev. B 78, 134109 (2008). 
[9] S. Gupta, S.C. Goyal. Sci China Ser D-Earth Sci. 52, 10, 1599 (2009).

[10] G.R. Barsch. J. Appl. Phys. 39, 3780 (1967).

[11] G.R. Barsch, Z.P. Chang. J. Appl. Phys. 39, 3276 (1968).

[12] R.C. Hollinger, G.R. Barsch. J. Phys. Chem. Solid 37, 845 (1976).

[13] V.K. Garg, D.S. Puri, M.P. Verma. Phys. Status Solidi 87, 401 (1978).

[14] K.P. Thakur. J. Phys. Chem. Solids 41, 465 (1979).

[15] E.V. Zarochentsev, S.M. Orel, V.N. Varyukhin. Phys. Status Solidi A 52, 455 (1979).

[16] E.V. Zarochentsev, S.M. Orel, V.N. Varyukhin. Phys. Status Solidi A 53, 75 (1979).

[17] E.V. Zarochentsev, S.M. Orel. Phys. Status Solidi A 57, 137 (1980).

[18] E.V. Zarochentsev, S.M. Orel, A.Yu. Yakovets. Phys. Status Solidi A 94, 515 (1986).

[19] E.V. Zarochentsev, S.M. Orel, I.V. Kochergin. Phys. Status Solidi A 94, 105 (1986).

[20] E.V. Zarochentsev, S.M. Orel, Yu.V. Stepanchuk. Phys. Status Solidi A 116, 295 (1989).

[21] E.V. Zarochentsev, V.N. Varyukhin, E.P. Troitskaya, V.V. Chabanenko, E.E. Horbenko. Phys. Status Solidi B 243, 12, 2672 (2006).

[22] В.Г. Барьяхтар, Е.В. Зароченцев, Е.П. Троицкая. Методы вычислительной физики в теории твердого тела. Атомные свойства металлов. Наук. думка, Киев (1990). 373 с.

[23] H. Shimizu, H. Tashiro, T. Kume, S. Sasaki, Phys. Rev. Lett. 86, 4568 (2001).

[24] B.M. Axilrod, E. Teller. J. Chem. Phys. 11, 299 (1943).

[25] W. Cochran. CPC Crit. Rev.Solid State Sci. 2, 1, 1 (1971).

[26] S. Gupta, S.C. Goyal. J. Phys. Chem. Solidi 64, 1125 (2003).

[27] M.P. Verma, R.K. Singh. Phys. Status Solidi 33, 769 (1969).

[28] К.Б. Толпыго. ЖЭТФ 20, 497 (1950).

[29] К.Б. Толпыго. УФЖ 2, 3, 242 (1957).

[30] К.Б. Толпыго. УФЖ 4, 72 (1959).

[31] К.Б. Толпыго. ФТТ 3, 4, 943 (1961).

[32] И.Г. Заславская, К.Б. Толпыго. УФЖ 1, 3, 226 (1956).

[33] 3.А. Демиденко, Т.И. Кучер, К.Б. Толпыго. ФТТ 3, 10, 2482 (1961).

[34] 3.А. Демиденко, К.Б. Толпыго. ФТТ 3, 11, 3435 (1961).

[35] К.Б. Толпыго, Е.П. Троицкая. ФТТ 13, 4, 1135 (1971).

[36] K.B. Tolpygo. Phys. Status Solidi B 56, 591 (1973).

[37] О.Н. Болонин, К.Б. Толпыго. ФТТ 15, 6, 1674 (1973).

[38] Е.П. Троицкая, В.В. Чабаненко, Е.Е. Горбенко. ФТТ 49, 11 , 2055 (2007).

[39] Е.П. Троицкая, В.В. Чабаненко, И.В. Жихарев, Е.Е. Горбенко. ФТТ 53, 8, 1555 (2011).

[40] Е.П. Троицкая, В.В. Чабаненко, И.В. Жихарев, Е.Е. Горбенко, Е.А. Пилипенко. ФТТ 54, 6, 1172 (2012).

[41] Е.П. Троицкая, В.В. Чабаненко, И.В. Жихарев, Е.Е. Горбенко, Е.А. Пилипенко. ФТТ 55, 2, 347 (2013).

[42] Е.П. Троицкая, В.В. Чабаненко, Е.А. Пилипенко, И.В. Жихарев, Е.Е. Горбенко. ФТТ 55, 11, 2218 (2013).

[43] V.N. Varyukhin, E.P. Troitskaya, Val.V. Chabanenko, Ie.Ie. Gorbenko, E.A. Pilipenko. Phys. Status Solidi B 251, 4, 774 (2014).

[44] V.N. Varyukhin, E.P. Troitskaya, Ie.Ie. Gorbenko, E.A. Pilipenko, V.V. Chabanenko. Phys. Status Solidi B 252, 709 (2015).

[45] F.D. Murnaghan. Finite deformation of clastic solids. John Wiley and Sons, N.Y. (1951) 68 p.

[46] D. Wallace. Solid State Phys. 25, 301 (1970).
[47] Е.В. Зароченцев, Е.П. Троицкая. ФТТ 43, 1292 (2001).

[48] M.S. Anderson, C.A. Swenson. J. Phys. Chem. Solidi 36, 145 (1975).

[49] H. Shimizu, N. Saitoh, S. Sasaki. Phys. Rev. B 57, 230 (1998).

[50] A.P. Jephcoat, H.K. Mao, L.W. Finger, D.F. Lox, R.J. Hemley, C.S. Zha. Phys. Rev. Lett. 59, 2, 2670 (1987).

[51] K.A. Goettel, J.H. Eggert, I.F. Silvera, W.C. Moss. Phys. Rev. Lett. 62, 665( 1989).

[52] C.A. Swenson. In: Rare Gas Solids. V. 11 / Eds M.L. Klein, J.A. Venables. Academic Press, N.Y. (1977). P. 863.

[53] C. Zener. Phes. Rev. 71, 12, 846 (1947).

[54] B.B. Karki, L. Stixrude, S.J. Clark, M.C. Warren, G.J. Ackland. Crain. J. Am. Miner 82, 51 (1997).

[55] F. Jona, P.M. Marcus. J. Phys.-Condens Matter 13, 5507 (2001).

[56] В.Д. Фараджев, З.Ф. Искендерзаде, Э.К. Касумова, Э.М. Курбанов. Неорган. материалы 41, 9, 1042 (2005).

[57] H. Shimizu, H. Imaeda, T. Kume, S. Sasaki. Phys. Rev. B 71, 014108 (2005).

[58] M. Grimsditch, P. Loubeyre, A. Polian. Phys. Rev. B 33, 7192 (1986).

[59] S. Sasaki, N. Wada, T. Kumi, H. Shimizu. J. Raman Spectroscopy 40, 121 (2009).

[60] A. Polian, J.V. Desson, M. Grimsditch, W.A. Grosshans. Phys. Rev. B 39, 2, 1332 (1989).

[61] T. Iitaka, T. Ebisuzaki. Phys. Rev. В 65, 012103 (2001).

[62] Е.П. Троицкая, И.В. Жихарев, Е.А. Пилипенко. ФТВД 24, 26 (2014). 